\title{
Arbor
}

\section{El hispanismo italiano en los umbrales del XXI (1998-2000): balance y perspectivas}

\section{Renata Londero}

Arbor CLXVIII, 664 (Abril 2001), 575-587 pp.

Los novísimos estudios hispánicos en Italia (1998-2000) se caracterizan por la heterogeneidad y amplitud de sus orientaciones, a pesar de seguir centrándose en el campo literario, y, dentro de él, en algunos períodos y autores preferidos ${ }^{1}$. No obstante, al lado de esta tendencia general se observa una cada vez más acusada apertura hacia otros ámbitos de investigación, como, especialmente, el lingüístico. En el preponderante marco literario, la variedad de las propuestas críticas - con la habitual predilección por el Siglo de Oro y el XX- se detecta fácilmente en recientes volúmenes misceláneos dedicados a maestros del iberismo italiano, como Mario Di Pinto (Signoria di parole, 1998) o Luciana Stegagno Picchio ( $E$ vós, Tágides minhas, 1999)2. En el homenaje a Di Pinto, el abanico es muy amplio: la canción castellana de Alfonso XI, Garcilaso, Cristóbal de Virués, Cervantes, Fernando de Herrera, las relaciones hispano-italianas en el teatro del XVII, los sonetos de Quevedo y de Góngora, las épocas dieciochescas y decimonónicas, y la poesía del siglo XX (Valle-Inclán, Gerardo Diego, García Lorca, Jorge Guillén, Rafael Alberti). Paralelamente, en el tomo en honor de Stegagno Picchio, las contribuciones de más estricto interés hispanístico conciernen al bilingüismo castellano-portugués en el Cancioneiro de Resende, la Celestina, las crónicas de la Conquista, Cervantes, la calderoniana Los tres mayores prodigios, Gracián y Portugal, los sonetos de Unamuno, y los poemas gallegos lorquianos ${ }^{3}$.

Con todo, quizás sea aún más significativo dirigir la mirada al abigarrado paisaje de los congresos, celebrados sea en centros académicos de antiguo prestigio hispanístico - Venecia, Milán, Bolonia, Pisa, Florencia, 
Roma, Nápoles, Palermo-, sea en universidades más pequeñas y jóvenes, pero con gran actividad, como Verona, Trento, Vercelli y Parma, entre otras. Para empezar, reviste indudable interés la extensión cronológica y temática que abarcan las comunicaciones leídas en el XVIII congreso de la Associazione Ispanisti Italiani (A.ISP.I., Siena, 5-7 de marzo de 1998, publ. 1999), titulado —en la sección literaria colo e scrittura: dal Medioevo ai giorni nostri. En la primera parte, reservada a las letras españolas ${ }^{5}$, al lado de corrientes, obras y autores bastante estudiados en Italia -la Celestina, el Persiles, Bécquer y Unamuno-, resaltan movimientos, géneros y figuras más descuidados: por lo que atañe a la Edad Media, se analizan Elena y María (Veronica Orazi) y la historiografía en latín de Rodrigo Ximénez de Rada (11701247; Andrea Zinato), mientras que a los libros de caballerías se dedica Anna Bognolo, una de las poquísimas expertas italianas de este género ${ }^{6}$; en la floresta de dramaturgos áureos menores Diego Símini escoge a Antonio Fajardo y Acevedo; y figuras de gran envergadura pero poco examinadas por el hispanismo italiano - Ganivet, Maeztu o Azorín-son objeto de análisis por parte de Giovanna Scalia, María José Flores y Francisco José Martín, respectivamente.

La multiplicidad también salta a la vista — si bien con unas claras preferencias - si se consideran otros congresos de ámbito hispánico en el trienio $1998-2000 .^{7} \mathrm{Si}$ se procede en orden cronológico en cuanto a las áreas de estudio, merece especial atención el simposio sobre la poesía cancioneril («Canzonieri iberici», Padua-Venecia, 25-27 de mayo del 2000) organizado por Patrizia Botta, experta de literatura española y portuguesa del XV. El fructífero sector de los vínculos culturales entre España e Italia durante los siglos XVI-XVII se ha explorado, por ejemplo, en «Spagna e Italia attraverso la letteratura del secondo Cinquecento» (Nápoles, 21-23 de octubre de 1999). Sobre la interpretación narrativa de la historia y de la experiencia en los siglos XV, XVI y XVII («Riscritture della narrativa-ss. XV-XVII») ha versado el encuentro internacional organizado por Giuseppe Grilli y Gianni Spallone (Nápoles-Cassino, 10-13 de mayo del 2000). Cervantes ha sido escudriñado en profundidad en simposios como «Cervantes e i generi letterari» (Vercelli, 28-29 de enero de 1998, organizador: José Manuel Martín Morán; publ. 2000) y la «VI Giornata Cervantina» de Padua (17-18 de abril de 1998, actas en prensa, a cargo de Donatella Pini y Carlos Romero Muñoz). Y en la época áurea se han centrado dos reuniones científicas de gran relieve: «Otro Lope no ha de haber» (Florencia, 10-13 de febrero de 1999), organizada por una de sus más profundas investigadoras, Maria Grazia Profeti (publ. 2000) ${ }^{8}$, y «Drammaturgia e spettacolarità nel teatro iberico dei Secoli d'Oro» 
El hispanismo italiano en los umbrales del XXI...

(Nápoles, 22-24 de abril de 1999), a cargo de Giovanni Battista De Cesare (publ. 2000).

Varios encuentros más han sido impulsados por aniversarios de realce, como los centenarios del 98 y del nacimiento de García Lorca. Recién publicadas están las actas de «Le conversazioni della vigilia. Il 98 al crocevia volontà/abulia» (Vercelli, 16-17 de mayo de 1997, organizadores: José Manuel Martín Morán y Giuseppe Mazzocchi), donde se sondean representativas facetas literario-filosóficas del pensamiento y la creación noventayochistas, con ponencias sobre el concepto de generación y la controvertida relación 98-Modernismo, o sobre autores específicos: Unamuno, Antonio Machado, Azorín. En este mismo ámbito también cabe señalar el congreso sobre Ortega y Gasset («Ortega narrador/pensador de Europa», Milán-Gargnano, 13-14 y 1618 de noviembre de 1998), organizado por Marco Cipolloni. A García Lorca, el poeta español más conocido y traducido en Italia, entre los meses de abril y mayo de 1998 se dedicaron una serie de congresos de alto nivel, a lo largo de nuestra península. Están en prensa las actas del «Incontro internazionále su Federico García Lorca» (Udine, 16-17 de abril de 1998, organizadores: Giancarlo Ricci y Luis Luque Toro; publ. 2000), donde críticos italianos, españoles, alemanes y eslovenos afrontan la obra del granadino desde la perspectiva de su «multimedialidad» artística. En Trento, Pietro Taravacci fue el promotor de «Un secolo di Lorca: tra Spagna e Italia» (23-24 de abril de 1998; publ. 2000), donde lorquistas de grueso calibre como Giovanni Caravaggi, Laura Dolfi, Mario Hernández, Piero Menarini, Gabriele Morelli, Andrés Soria Olmedo y Norbert von Prellwitz debatieron aspectos intertextuales e interdiscursivos en la producción de Federico. Una mención especial merece el congreso organizado por Laura Dolfi en Parma ( Federico García Lorca e il suo tempo», 27-29 de abril de 1998; publ. $1999^{9}$ ), al que acudieron prestigiosos lorquistas y expertos del 27 -A. Soria Olmedo, Enric Bou, James Valender, José Carlos Rovira, Christian de Paepe, María Clementa Millán, entre otros-, para explorar los múltiples lazos culturales entre Lorca y escritores españoles, latinoamericanos y sajones. Complementa las actas un apéndice a cargo de Laura Dolfi, donde tras una exhaustiva reseña bibliográfica sobre la fortuna crítica lorquiana hasta 1946, destacan las inéditas y valiosas versiones italianas del teatro lorquiano ofrecidas por Oreste Macrí (Amor de don Perlimplín y Belisa en su jardín, Retablillo de don Cristóbal, La zapatera prodigiosa, Bodas de sangre, El maleficio de la mariposa). Cierran este rico conjunto lorquiano los coloquios de Bolonia (organizador: Piero Menarini), «Studiosi italiani di 
fronte a Lorca» (4 de mayo de 1998), y de Palermo (a cargo de Maria Caterina Ruta), «Dal 98 a García Lorca: lacerazioni e strutture della cultura spagnola del 900» (11-12 de mayo de 1998). Aparte de Federico, otros poetas del 27 han disfrutado de una buena acogida: es el caso de Rafael Alberti, a quien se dedican las bellas páginas de Ripensando a Rafael Alberti (1999), actas de la homónima jornada de estudios (Anticoli Corrado, 16 de mayo de 1998). Y finalmente, dos interesantísimos contemporáneos de Alberti, mucho menos estudiados en Italia, Manuel Altolaguirre y José Bergamín, se homenajearon en sendos coloquios: «Manuel Altolaguirre y las revistas literarias de la época» (Bérgamo, 5-6 de mayo de 1997, a cargo de G. Morelli, publ. 1999), y «José Bergamín, tra avanguardia e barocco» (Verona, 2-4 de abril de 1998, organizadora: Paola Ambrosi; publ. 2000).

Para concluir, cito sólo tres títulos modélicos de posibles rumbos que el hispanismo italiano podría tomar en el futuro. Un imprescindible movimiento cultural como el Romanticismo, todavía poco aprovechado en Italia por lo que se refiere a su vertiente española, se investiga, por iniciativa de Ermanno Caldera y Piero Menarini, en Romanticismo 7. La poesía romántica, Actas del VII Congreso del Centro Internacional de Estudios sobre el Romanticismo hispánico (Nápoles, 23-25 de marzo de 1999; publ. 2000). La creciente afición por los cruces interdiscursivos y la interdisciplinaridad promete aplicarse también a los estudios hispánicos en Italia: un ejemplo al respecto son las actas (1999) del congreso sobre la autobiografía en España e Hispanoamérica, a cargo de Rosalba Campra y Norbert von Prellwitz, Le scritture dell'io (Roma, 22-23 de noviembre de 1996). Por otra parte, el productivo gusto por las intersecciones entre lenguaje verbal y figurativo sale al descubierto en el tema del XIX congreso de A.ISP.I. (sección literaria; Roma, 16-18 de septiembre de 1999; actas en prensa), «Le arti figurative nelle letterature iberiche».

Si se pasa a examinar las monografías y los artículos, de nuevo se nota la fusión de tradición e innovación en la elección de períodos, autores y temas: el Siglo de Oro sigue suscitando un poderoso atractivo, aunque se perfila un interés cada vez mayor por campos hasta ahora menos excavados, como el Realismo y la novela de la primera mitad del XX. En un espigueo muy parcial se pueden destacar las sutiles anotaciones de Gaetano Chiappini en su libro sobre los místicos (1999); el atinado ensayo de Mariarosa Scaramuzza (1998) sobre la utopía en el Quijote y la dialéctica imaginación/deseo en el Persiles; el libro de Alessandro Martinengo sobre el Marco Bruto de Quevedo (1998). En cuanto a caminos novedosos, hay que subrayar, por ejemplo, la atención que Maria Grazia Profeti (1998) y Elide Pittarello (1999) reservan a un personaje extraordinario y desechado como 
Torres Villarroel. La novela del XIX y del XX también ha sido objeto de fecundas indagaciones, con estudios sobre escritores no muy aprovechados por nuestro hispanismo: léanse el libro de Assunta Polizzi sobre la metaficción en Galdós (1999), el de Carla Prestigiacomo acerca de la novelística de Gómez de la Serna (1999), y las monografías de Maria Rosaria Alfani sobre Clarín (2000), o de Luisa Chierichetti sobre el humor en la narrativa de López Rubio, Jardiel Poncela y Neville (1999).

Igualmente, clásicos y modernos, grandes y pequeños, predilectos y descuidados se entrecruzan en el sector ecdótico hispánico, respaldado por la prestigiosa tradición filológica italiana ${ }^{10}$. Aún en una selección muy restringida cabe realzar la edición crítica de los Proverbios morales de Sem Tob (1998) a cargo de Marcella Ciceri, que también tiene en preparación una edición del Libro de Buen Amor; la edición bilingüe del Doctrinal de gentileza de Hernando de Ludueña por uno de los mejores jóvenes filólogos hispánicos italianos, Giuseppe Mazzocchi (1998), la fatiga ecdótica de Aldo Ruffinatto sobre el Lazarillo (2000), la impecable edición de La selva sin amor lopiana por Maria Grazia Profeti $(1999)^{11}$, la Dama duende calderoniana, editada con gran esmero por Fausta Antonucci (1999). Otro joven y muy válido filólogo hispánico, Stefano Arata, tiene en prensa (Madrid, Castalia) la edición de El acero de Madrid de Lope. A estas iniciativas es preciso añadir por lo menos «agua y peña», la importante colección de textos ibéricos, antiguos, inéditos o raros de los siglos XVI y XVII, nacida en 1997 bajo la dirección de Giovanni Caravaggi. En 1998 y 1999 han visto la luz la edición de la fiesta teatral burlesca Las bodas de Orlando (1685) (Javier Huerta Calvo), las Fábulas mitológicas de Cristóbal de Castillejo (Blanca Periñán), la comedia burlesca de Jerónimo de Cáncer y Juan Vélez de Guevara, Los siete infantes de Lara (Pietro Taravacci), e "Història de la filla del rei d'Hungria» e altri racconti catalani tardomedievali (Veronica Orazi). En el 2000 se han publicado la Historia de Grisel y Mirabella de Juan de Flores (Maria Grazia Ciccarello), el poema alegórico de Luis Hurtado de Toledo, Hospital de necios (Valentina Nider y Ramón Valdés), La historia de Griselda (c. 1544) (Juan Carlos Conde y Víctor Infantes), dos comedias de enredo de finales del XVII -De la noche a la mañana de Ceferino Clavero de Falces (Olga Perotti), y No puede mentir el cielo de Andrés Gil Enríquez (Renata Londero) - y dos textos festivos de Quevedo, la Vida de la Corte y las Capitulaciones matrimoniales (Marcial Rubio Árquez). Si, para terminar, nos acercamos al siglo XX, entre las muchas ediciones recientes se pueden recordar las de Diario de un enfermo (ed. Francisco José Martín Cabrero, 2000) y de El maleficio de la mariposa (ed. P. Menarini, 1999), porque representan el interés hacia dos autores como Azorín y García Lorca, tan 


\section{Renata Londero}

distanciados en la consideración crítica y lectora italiana, al estar el primero casi olvidado ${ }^{12}$, y el segundo en la cumbre del renombre.

El hispanismo italiano, no obstante, no se (auto)dirige sólo a una élite de especialistas, sino que - con ahínco cada vez mayor- se esfuerza por difundir la apreciación de la cultura hispánica en más vastos círculos de lectores. En un nivel de alta divulgación, por ejemplo, se colocan manuales como la historia de la literatura española en varios tomos coordinada por Maria Grazia Profeti, de la que han salido los volúmenes sobre el XVI y el XVII (1998), y el XVIII-XIX (1999). Y por supuesto un lugar de relieve lo ocupan las traducciones. Citando a Lore Terracini, «professionisti dell'ispanismo", «letterati e poeti», pero también "persone di cultura» ${ }^{13}$ podrán disfrutar de las versiones italianas de clásicos antiguos o modernos salidas recientemente. Por lo que concierne a la Edad Media, son de señalar los Miracoli della Vergine bilingües editados por Carlo Beretta (1999), donde aparecen todos los Milagros de Berceo y una amplia selección de las Cantigas de Santa María de Alfonso el Sabio; y la buena traducción del Libro de buen amor por Vincenzo La Gioia (1999), enriquecida por la introducción, la anotación y la bibliografía a cargo de Giuseppe Di Stefano. Del inmenso mar de las letras áureas han vuelto a aflorar algunas joyas: la Lozana andalu$z a$ (traducida con gran acierto por Teresa Cirillo en 1998), la mística (v. la traducción del Castillo de perfección por Angelo Morino, 1999), la comedia (v. la edición italiana del Burlador de Sevilla por Laura Dolfi, 1998) y la preceptiva teatral (v. la nueva edición bilingüe del Arte nuevo de hacer comedias por Maria Grazia Profeti, 1999). Pasando al XX, el lector puede deleitarse con el transgresivo carnaval valleinclanesco de Las galas del difunto (trad. de Paola Ambrosi, 1999), para después sumergirse en las Soledades machadianas, traducidas por varios poetas italianos y portugueses en la antología de Giancarlo Depretis (2000). Más éxito editorial está cosechando en Italia la novela española de las dos últimas décadas. Por ejemplo, es de 1999 la traducción de Corazón tan blanco de Javier Marías, y en el 2000 ha salido L'uomo sentimentale (El hombre sentimental). De la afortunada narrativa de Antonio Muñoz Molina acaban de salir en italiano Los misterios de Madrid (1998) y su «opera prima» Beatus ille (1999); Lo raro es vivir de Carmen Martín Gaite se tradujo en 1998 y la versión italiana de Irse de casa es muy reciente (2000); en el 2000 también ha sido traducido a nuestra lengua Olvidado rey Gudú de Ana María Matute. Con miras puramente comerciales, en la estela de éxitos más o menos transgresivos en España, se han traducido novelas como La tempestad de Juan Manuel de Prada (1998), o Beatriz y los cuerpos celestes de Lucía Etxebarría (1999). La cantidad de traducciones -no desdeñable por lo que atañe a España, aunque todavía mínima si se compara con el mundo anglosajón o francófono- au- 
El hispanismo italiano en los umbrales del XXI...

menta considerablemente si se tiene en cuenta, en cambio, la narrativa (y, pero mucho menos, la poesía) hispanoamericana contemporánea: para los lectores italianos de hoy la gama es bastante vasta, de Borges a Neruda, de Vargas Llosa a Octavio Paz, de Mario Benedetti a Luis Sepúlveda ${ }^{14}$.

Bien es verdad que mucho queda por hacer, aunque algo se está moviendo en direcciones esperanzadoras. Como hemos visto, la literatura continúa ejerciendo su primacía en nuestro hispanismo, pero despiertan intereses nuevos. Nacen revistas (1998) como la anual «Rivista di Filologia e Letterature Ispaniche», coordinada por Giuseppe Di Stefano en Pisa; o surgen iniciativas importantes como la creación del «Centro Interdipartimentale di Studi sulla Lombardia Spagnola», dirigido por Giovanni Caravaggi en la Universidad de Pavia. Y las tesis doctorales discutidas en las sedes de Bolonia, Pisa y Palermo en $1999^{15}$ demuestran un constante y eficaz aprovechamiento de las letras áureas (v. las ediciones críticas de la Fama póstuma de Pérez de Montalbán, por Enrico Di Pàstena, o de La Hermosura de Angélica de Lope, por Marcella Trambaioli), al lado de inéditas indagaciones sobre el teatro español del primer tercio del XX (Floriana Di Gesù) o la narrativa de hoy (Luigi Contadini, La scrittura ambivalente di Juan José Millás).

Otras señales de cambio van apuntando fuera de la esfera literaria: paralelamente a la ampliación de la enseñanza del español en nuestras universidades destaca la importancia que están cobrando los estudios lingüísticos. En 1999 proliferaron congresos de relieve: se ocuparon de la relación contrastiva italiano/español los estudiosos que intervinieron en la sección de lengua del citado XIX congreso de A.ISP.I («Italiano e spagnolo a contatto»), mientras que problemas más bien vinculados con la traducción y la glotodidáctica se discutieron en los congresos «Creación de materiales y nuevas tecnologías» (Milán, 25-26 de febrero de 1999, publ. 2000) e «Interpretar Traducir Textos de la(s) cultura(s) hispánica(s)» (Forlì, 21-23 de octubre de 1999 , actas en prensa). A nivel institucional una novedad de notable peso es la puesta en marcha (2000) del primer Doctorado de Lingüística Hispánica (dentro del «Dottorato di Linguistica delle Lingue Moderne») coordinado por Encarnación García Dini en la Universidad de Pisa. Y finalmente, el panorama de los cursos de español para italianos (hasta hace pocos años casi desierto) también se innova: en 1998 salió Amigo sincero, de Maria V. Calvi y Nicelda Provoste, y en el prometedor campo de los lenguajes sectoriales se encauzan $\dot{i}$ A cómo está la lira? (1998) de Luis Luque Toro y Giancarlo Ricci, y el curso multimedia de Félix San Vicente, En este país (1999).

Hemos llegado a la meta de este trayecto fugaz y somero por los recovecos del hispanismo italiano de hoy: de punto final nos pueden servir los significativos títulos de las secciones literaria y lingüística previstas para 
el primer congreso de la Associazione Ispanisti Italiani en el siglo XXI (Florencia, 15-17 de marzo del 2001): «La penna di Venere. Scritture dell'amore nelle letterature iberiche»-«Testi specialistici e nuovi saperi nelle lingue iberiche». Cómo armonizar tradición e innovación, lo universal y lo particular, es otra vez el reto asignado a los hispanistas italianos del inmediato porvenir ${ }^{16}$.

\section{Notas}

1 Para un excursus del hispanismo italiano a partir de comienzos del XX, v. las actas del congreso de A.ISP.I (Associazione Ispanisti Italiani) L'apporto italiano alla tradizione degli studi ispanici-Nel ricordo di Carmelo Samonà (Nápoles, 30-31 de enero, 1 de febrero de 1992), Roma, Instituto Cervantes, 1993; la introducción (pp. 9-23) y la parte II («Tradurre letteratura», pp. 173-224) del libro de Maria Grazia Profeti, Importare letteratura: Italia e Spagna, Alessandria, Dell'Orso, 1993; y los artículos de Laura Dolfi («Para una bibliografía del siglo XVII-Prosa y poesía: 1980-1989», en Los estudios hispánicos en Italia y en Alemania. Estado presente de la investigación y perspectivas para el futuro, Actas del congreso de Villa Vigoni (21-23 de mayo de 1989), R. Froldi y M. Tietz (eds.), Bochum, Ruhr-Universität, 1993, pp. 59-72); Lore Terracini («Tradurre letteratura spagnola in Italia», en El Girador. Studi di Letterature Iberiche e Ibero-americane offerti a Giuseppe Bellini, G.B. De Cesare y S. Serafin (eds.), Roma, Bulzoni, 1993, t. II, pp. 1015-1023); Aldo Ruffinatto ("L'ispanistica italiana”, en Gli spagnoli e l'Italia, D. Puccini (ed.), Milano, Scheiwiller, 1997, pp. 125-130). Noticias pormenorizadas sobre los frutos colectivos del hispanismo italiano, evidentes en las actas de los congresos de A.ISP.I desde su fundación (1973) hasta 1997, se pueden hallar en L. Dolfi, Storia dell'A.ISP.I (Associazione Ispanisti Italiani) 1973-1997, Roma, Bulzoni, 1998. Finalmente, son fundamentales el Repertorio bibliografico degli Ispanisti Italiani 1992, a cargo de Paola Elia (Chieti, Università «G. D’Annunzio», 1993), y el Repertorio bibliografico degli Ispanisti Italiani-Integrazione (fino al 1992) e Aggiornamento (1993-1996), Antonella Cancellier y Luisa Selvaggini (eds.), Roma, Bulzoni, 1998. Está en preparación la puesta al día del Repertorio, con las publicaciones del trienio 1996-1999, de la que se encargarán A. Cancellier, Renata Londero y L. Selvaggini.

2 Todos los libros y artículos citados de forma abreviada en el texto quedan consignados en la Bibliografía final.

3 En los Noventa se han editado otros importantes homenajes a grandes hispanistas italianos, que por motivos de límites cronológicos no analizo detenidamente en este trabajo. Cabe, sin embargo, al menos citarlos a continuación. A Giuseppe Bellini están dedicadas dos obras misceláneas: el mencionado El Girador (1993, 2 tt.), y Un lume nella notte. Studi di Iberistica che allievi e amici dedicano a Giuseppe Bellini, S. Serafin (ed.), Roma, Bulzoni, 1997. Para homenajear a Ermanno Caldera en 1993 se publicó De místicos y mágicos, clásicos y románticos, A. Albonico, G. Bellini, A. Calderone, R. Froldi, E. Pittarello (eds.), Messina, Armando Siciliano Editore; mientras que Lore Terracini fue conmemorada en Una giornata per Lore. Ricordo di Lore Terracini nel primo anniversario della sua scomparsa, A. Ruffinatto, A. Morino, M. Cipolloni, M. Rosso Gallo (eds.), Torino, Istituto dell'Atlante Linguistico Italiano, 1997. Y finalmente, en 1996 salieron los dos relevantes 


\section{El hispanismo italiano en los umbrales del XXI...}

tomos de Studi Ispanici de Oreste Macrí (Napoli, Liguori), donde Laura Dolfi recogió una amplia selección de ensayos de este maestro del hispanismo italiano, centrados en escritores y críticos españoles e hispanoamericanos (t. I-Poeti e Narratori; t. II-I critici).

4 La sección lingüística se tituló Lo spagnolo d'oggi: forme della comunicazione y acogió 14 comunicaciones.

5 En el tomo aparecen también una pequeña sección de literatura catalana, y una parte más amplia dedicada a las letras hispanoamericanas.

6 De la misma autora es el libro La finzione rinnovata. Meraviglioso, corte e avventura nel romanzo cavalleresco del primo Cinquecento spagnolo, Pisa, ETS, 1997.

7 Por razones de espacio voy a proporcionar sólo una pequeña selección de los muchos congresos de interés hispanístico que han tenido lugar en Italia en los últimos tres años, privilegiando los encuentros cuyas actas están publicadas o en prensa.

8 Las riquísimas actas (con secciones dedicadas a documentos inéditos, a la novela, la poesía y el teatro del Fénix, a sus modelos literarios y a sus enlaces intertextuales con autores -sobre todo españoles e italianos- desde el siglo XVII hasta nuestros días, constituyen los números 14, 15 y 16 de la colección "Secoli d'Oro" dirigida por M.G. Profeti y Gaetano Chiappini en la editorial florentina Alinea, donde hasta hoy se han recogido interesantes volúmenes misceláneos, actas de seminarios, monografías y antologías sobre todo centrados en el teatro, la novela y la poesía auriseculares.

9 Con estas actas se inaugura la colección de ensayos (dedicados a temas de filología hispánica comparada) «Euro-ispanica», dirigida por L. Dolfi en la editorial Bulzoni.

10 Cf. Aldo Ruffinatto, Ispanismo e ecdotica, en A.ISP.I, L'apporto italiano..., cit., pp. 9-21; y Alberto Vàrvaro, Ispanismo e filologia romanza, íbid, pp. 33-42.

${ }^{11}$ La edición está incluída en la colección de textos y ensayos "Barataria», dirigida por L. Dolfi y M. Di Pinto en la editorial napolitana Liguori.

12 En los últimos treinta años en Italia ha salido sólo un libro sobre Azorín: Renata Londero, Nell'officina dello scrittore. I romanzi di Azorín fra gli anni Venti e Quaranta, Padova, Unipress, 1997.

13 Tradurre letteratura spagnola in Italia, en El Girador, cit., p. 1016.

14 Cito sólo una recortadísima muestra de textos hispanoamericanos traducidos en Italia en el último trienio, que puede dar suficiente cuenta del interés de un público tanto especialista como amateur por este complejo mundo cultural. En la editorial Adelphi está saliendo la obra completa de Jorge Luis Borges, a cargo de Antonio Melis, Fabio Rodríguez Amaya y Tommaso Scarano (Laboratorio Borges): cf. L'Aleph (1998), Testi prigionieri (1998), L'artefice (1999), a cargo de T. Scarano; Il manoscritto di Brodie (1999, a cargo de A. Melis). En 1999, Einaudi publicó Evaristo Carriego (a cargo de Paolo Collo y Jaime Riera Rehen). Numerosas han sido las versiones italianas de Neruda: Il fromboliere entusiasta (trad. R. Bovaia), Parma, Guanda, 1998; Memoriale di Isla Negra (a cargo de Giuseppe Bellini), Firenze, Passigli, 1998. En la misma editorial (y a cargo de G. Bellini) han salido también Residenze sulla terra (1999), Venti poesie d'amore e una canzone disperata (1999), Duemila (1999), Fine del mondo (2000). Otros muchos autores chilenos son conocidos por el público italiano: entre ellos, Isabel Allende (en 1999 Elena Liverani tradujo La hija de la fortuna, Milano, Feltrinelli), Luis Sepúlveda (en 1999 salió la traducción de Jacaré, Parma, Guanda), Francisco Coloane (L'ultimo mozzo della Baquedano, ivi, 2000), Marcela Serrano (L'albergo delle donne, Milano, Feltrinelli, 1999; Il tempo di Blanca, ivi, 2000). En cuanto al cercano Uruguay, hay que señalar por lo menos la traducción de cuentos de Mario Benedetti, cuidada por Martha Canfield (Segnali di fumo, trad. de Emanuela Jossa, Firenze, Le Lettere, 2000), y la doble versión italiana de Ariel de José Enrique Rodó (a car- 
go de A. Cancellier, Bologna, "In forma di parole», 1999; a cargo de M. Canfield, trad. de D. Símini, Firenze, Alinea, 2000). Tres celebridades hispanoamericanas siguen traduciéndose con frecuencia: Gabriel García Márquez (Gente di Bogotá 1954-1955, trad. it. de A. Morino, Milano, Mondadori, 1999), Mario Vargas Llosa (Lettere a un aspirante romanziere, Torino, Einaudi, 1998), y Octavio Paz (Vento cardinale e altre poesie, a cargo de Franco Mogni, Milano, Mondadori, 1999). Es casi obligatoria la referencia al muy reciente boom de las letras cubanas en nuestro país: por ejemplo, Danilo Manera, ferviente promotor de la cultura de la isla caribeña en Italia, continúa dando a conocer nuevas voces de su narrativa en Rumba senza palme né carezze. Racconti di donne cubane (Milano, Feltrinelli, 1999). Además, v. la antología bilingüe La terra delle mille danze. Il racconto cubano oggi, edición de Francisco López Sacha y traducida por Ignazio Delogu (Firenze, Ibiskos, 1999), y "Cubalibri», la colección de traducciones de autores cubanos coordinada por Diego Símini, de la que ha salido el primer número, Prosas leves (Prose lievi) de Cintio Vitier (edición de D. Símini, Lecce, Fondamentale, 2000).

15 Cf. L. Dolfi, El doctorado en Italia, en Doctorat et recherches dans l'hispanisme européen. Actes des Journées d'études de Paris et Saint-Jacques de Compostelle (9-12 mai 1996), É. Lavaud-Fage et M. Moner (eds.), Tours, Societé des Hispanistes Français de l'Enseignement Supérieur, 1998, pp. 59-72.

16 Deseo expresar mi sincero agradecimiento a todos los amigos y colegas que con sus informaciones, consejos y envíos de material han facilitado la labor de recolección bibliográfica necesaria para este artículo: Paola Ambrosi, Fausta Antonucci, Patrizia Botta, Ermanno Caldera, Maria Vittoria Calvi, Antonella Cancellier, Martha Canfield, Marco Cipolloni, Giovanni Battista De Cesare, Giancarlo Depretis, Laura Dolfi, Giuseppe Grilli, Augusto Guarino, Francisco Lobera Serrano, Luis Luque Toro, Francisco José Martín Cabrero, José Manuel Martín Morán, Giuseppe Mazzocchi, Piero Menarini, Elide Pittarello, Assunta Polizzi, Maria Grazia Profeti, Aldo Ruffinatto, Mariarosa Scaramuzza, Diego Símini, Pietro Taravacci, Norbert von Prellwitz.

\section{Bibliografía consultas}

\section{Tomos misceláneos}

E vós, Tágides minhas. Miscellanea in onore di Luciana Stegagno Picchio, M. J. de Lancastre, S. Peloso, U. Serani (eds.), Lucca-Viareggio, Mauro Baroni, 1999.

Signoria di parole. Studi offerti a Mario Di Pinto, G. CALABrò (ed.), Napoli, Liguori, 1998.

\section{Actas de congresos (publicadas)}

A.ISP.I, XVIII Congreso, Fine secolo e scrittura: dal Medioevo ai giorni nostri - Lo spagnolo d'oggi: forme della comunicazione (Siena, 5-7 de marzo de 1998), Roma, Bulzo$\mathrm{ni}, 1999,2$ tt.

Equipo Aula Cervantes, Creación de materiales y nuevas tecnologías (Milán, 25-26 de febrero de 1999), Lucca-Viareggio, Mauro Baroni, 2000.

Cervantes e i generi letterari (Vercelli, 28-29 de enero de 1998), J. M. Martín Morán (ed.), Vercelli, Mercurio, 2000.

Dal testo alla scena. Atti del convegno "Drammaturgia e spettacolarità nel teatro iberico dei Secoli d'Oro» (Nápoles, 22-24 de abril de 1999), G.B. De Cesare (ed.), Salerno, Il Paguro, 2000. 


\section{El hispanismo italiano en los umbrales del XXI...}

Federico García Lorca e il suo tempo, Atti del Congresso internazionale (Parma, 27-29 de abril de 1998), L. Dolfi (ed.), Roma, Bulzoni, 1999.

Incontro internazionale su Federico García Lorca (Udine, 16-17 de abril de 1998), G. Ricci, L. Luque Toro (eds.), Udine, Forum, 2000.

José Bergamín, tra avanguardia e barocco (Verona, 2-4 de abril de 1998), P. Ambrosi (ed.), Pisa, ETS, 2000.

Le conversazioni della vigilia. Il 98 al crocevia volontà / abulia (Vercelli, 16-17 de mayo de 1997), J. M. Martín Morán, G. Mazzocchi (eds.), Lucca-Viareggio, Mauro Baroni, 2000.

Manuel Altolaguirre y las revistas literarias de la época (Bérgamo, 5-6 de mayo de 1997), G. Morelli (ed.), Lucca-Viareggio, Mauro Baroni, 1999.

Ortega narrador de Europa, Ortega pensador de Europa (Milán-Gargnano, 13-14 y 16-18 de noviembre de 1998), M. Cipolloni (ed.), Napoli, Liguori, 2000.

"Otro Lope no ha de haber» (Florencia, 10-14 de febrero de 1999), M. G. Profeti (ed.), Firenze, Alinea, 2000, 3 tt.

Ripensando a Rafael Alberti, Atti del convegno di studi (Anticoli Corrado, 16 de mayo de 1998), M. C. Desiderio, F. J. Lobera Serrano, M. Zagolin, (eds.), prólogo de O. Lottini, Gaeta, Bibliotheca, 1999.

Romanticismo 7. La poesía romántica, Actas del VII Congreso del Centro Internacional de Estudios sobre el Romanticismo hispánico (Nápoles, 23-25 de marzo de 1999), P. Menarini (ed.), Bologna, Il Capitello del Sole, 2000.

Le scritture dell'io (Roma, 22-23 de noviembre de 1996), R. Campra, N. von Prellwitz (eds.), Roma, Il Bagatto, 1999.

Un secolo di Lorca: tra Spagna e Italia (Trento, 23-24 de abril de 1998), P. Taravacci (ed.), Trento, Editrice dell’Università degli Studi di Trento-Dipartimento di Scienze Filosofiche e Storiche, 2000.

\section{Monografias}

Alfani, MARIA Rosaria, Il ritorno di don Chisciotte. Uno studio su Clarín e le origini della letteratura spagnola contemporanea, Roma, Donzelli, 2000.

Chiappini, Gaetano, Esperienze di mistica spagnola: S. Teresa d'Avila, S. Giovanni della Croce e S. Ignazio di Loyola, Firenze, Alinea, 1999.

CHIERICHeTTI, LUISA, Narrazione e umorismo: $i$ romanzi di anteguerra di López Rubio, Jardiel Porcela e Neville, Milano, Mondini, 1999.

Martinengo, Alessandro, El Marco Bruto de Quevedo. Una unidad en dinámica transformación, Bern, Lang, 1998.

Polizzi, Assunta, El proceso metafictivo en el realismo de Pérez Galdós, Las Palmas de Gran Canaria, Ediciones del Cabildo Insular, 1999.

Prestigiacomo, CARLA, L'utopia dell'immortalità nei romanzi di Ramón Gómez de la Serna, Palermo, Edizioni della Fondazione Nazionale «Vito Fazio-Allmayer», 1999.

Scaramuzza, Mariarosa, Deseo, imaginación, utopía en Cervantes, Roma, Bulzoni, 1998.

\section{Ediciones críticas}

Anónimo, Una fiesta burlesca del siglo de oro. Las bodas de Orlando (Comedia, Loa y Entremeses), ed. Javier Huerta Calvo, Lucca-Viareggio, Mauro Baroni, 1998. 
Calderón de la Barca, Pedro, La dama duende, edición, prólogo y notas de Fausta Antonucci, estudio preliminar de Marc Vitse, Barcelona, Crítica, 1999.

Cáncer, JerónImo DE-VÉlez DE Guevara, JuAn, Los siete infantes de Lara, ed. Pietro Taravacci, Lucca-Viareggio, Mauro Baroni, 1999.

Castillejo, Cristóbal de, Fábulas mitológicas, ed. Blanca Periñán, Lucca-Viareggio, Mauro Baroni, 1999.

Clavero de Falces, Ceferino, De la noche a la mañana, ed. Olga Perotti, Lucca-Viareggio, Mauro Baroni, 2000.

FloReS, JUAN DE, Historia de Grisel y Mirabella, ed. Maria Grazia Ciccarello, Lucca-Viareggio, Mauro Baroni, 2000

García LorCa, Federico, El maleficio de la mariposa, ed. Piero Menarini, Madrid, Cátedra, 1999.

Gil EnRíquez, ANDRÉs, No puede mentir el cielo, ed. Renata Londero, Lucca-Viareggio, Mauro Baroni, 2000.

La historia de Griselda (c. 1544), eds. Juan Carlos Conde y Víctor Infantes, Lucca-Viareggio, Mauro Baroni, 2000.

Història de la filla del rei d'Hungria e altri racconti catalani tardomedievali, ed. Veronica Orazi, Lucca-Viareggio, Mauro Baroni, 1999.

Hurtado de TOLEdo, Luis, Hospital de necios, eds. Valentina Nider y Ramón Valdés, Lucca-Viareggio, Mauro Baroni, 2000.

LOPE DE VEGA, La selva sin amor, ed. Maria Grazia Profeti, Firenze, Alinea, 1999.

LudueÑa, HeRnANDO DE, Doctrinal de gentileza, ed. Giuseppe Mazzocchi, Napoli, Liguori, 1998.

Martínez Ruiz, José/Azonín, Diario de un enfermo, ed. Francisco José Martín Cabrero, Madrid, Biblioteca Nueva, 2000.

Quevedo, Francisco DE, Vida de la Corte y oficios entretenidos en ella. Capitulaciones matrimoniales, ed. Marcial Rubio Árquez, Lucca-Viareggio, Mauro Baroni, 2000.

RuffinatTo, Aldo, Las dos caras del Lazarillo: texto y mensaje, Madrid, Castalia, 2000.

Sem Tob de Carrión, Proverbios morales, ed. Marcella Ciceri, Modena, Mucchi, 1998.

\section{Artículos}

Pittarello, Elide, «La vita materiale di Diego de Torres Villarroel», Rassegna Iberistica, 67, 1999, pp. 3-16.

Profeti, Maria Grazia, «I Juguetes de Talía di Torres Villarroel: un itinerario "borghese"", en M.G. Profeti, E. García Dini, N. Guasti, M. Lombardi, I secoli d'oro e i lumi. Processi di risemantizzazione, Firenze, Alinea, 1998, pp. 9-22.

\section{Manuales de historia literaria}

VV.AA., L'età d'oro della letteratura spagnola. Il Cinquecento, a cura di M.G. Profeti, Firenze, La Nuova Italia, 1998.

VV.AA., L'età d'oro della letteratura spagnola. Il Seicento, a cura di M.G. Profeti, Firenze, La Nuova Italia, 1998.

VV.AA., L'età moderna della letteratura spagnola. Il Settecento e l'Ottocento, a cura di M.G. Profeti, Firenze, La Nuova Italia, 1999. 


\section{El hispanismo italiano en los umbrales del XXI...}

\section{Cursos de lengua española}

Calvi, Maria Vittoria-Provoste, Nicelda, Amigo sincero. Curso de español para italianos, Bologna, Zanichelli, 1998.

LUQUe TORO, LUIS-RICCI, GIANCARLo, ¿A cómo está la lira? Curso teórico-práctico de español económico para italianos, Udine, Forum, 1998.

San Vicente, FÉlix, En este país. El español de las ciencias sociales (libro+CD-Rom), Università degli Studi di Bologna-Sede di Forlì, CLUEB, 1999.

\section{Traducciones}

Delicado, Francisco, La lozana andaluza, a c. di T. Cirillo Sirri, Roma, Roma nel Rinascimento, 1998.

EtXebarRíA, LucíA, Beatriz e $i$ corpi celesti, Parma, Guanda, 1999.

LoPe DE VEGA, Nuova arte di far commedie in questi tempi, a c. di M. G. Profeti, Napoli, Liguori, 1999.

Machado, Antonio, Soledades. Solitudini. Saudades. Poesie spagnole tradotte da poeti italiani e portoghesi di Machado, a c. di G. Depretis, Alessandria, Dell'Orso, 2000.

MARÍAS, JAVIER, Un cuore così bianco, trad.it. de B. Lazzaro, Roma, Donzelli, 199sLa tempesta, trad. it. de S. Cherchi, Roma, e/o, 1998.

Ruiz, JuAN, ARcipreste DE HitA, Libro del Buon Amore, trad. it. de V. La Gioia, introd., notas y bibliografía de G. Di Stefano, Milano, Rizzoli, 1999.

Teresa De Jesús, Il castello interiore, a c. di A. Morino, Palermo, Sellerio, 1999.

Tirso De Molina, L'ingannatore di Siviglia, cura e trad. di L. Dolfi, Torino, Einaudi, 1998.

VAlle Inclán, RAmón Del, Il defunto va di gala, a c. di P. Ambrosi, Pisa, ETS, 1999. 